\title{
Modelling autoimmune lung disease
}

The discovery of a lung-specific autoantigen, the loss of tolerance to which can result in autoimmunemediated lung disease, has provided an explanation for the common pulmonary complications of autoimmune diseases.

Systemic autoimmune diseases such as rheumatoid arthritis are often complicated by interstitial lung disease (ILD), involving fibrosis and inflammation in the lung parenchyma. ILD can also occur spontaneously in the absence of autoimmune disease. Despite indirect evidence that both forms of ILD are autoimmune responses, until now there has been no conclusive identification of a pathogenic autoantigen.

Humans with mutations in the autoimmune regulator (AIRE) gene develop autoimmune polyendocrine syndrome type 1 ( $\underline{\text { APS1}}$ ), which can involve the lungs in some patients.

Anderson and colleagues showed that Aire I- $^{-/}$

$\mathrm{BALB} / \mathrm{c}$ and non-obese diabetic (NOD) mice develop a similar cellular interstitial pneumonia to the pulmonary disease of patients with APS1. In both cases, the cellular infiltrate consisted mainly of $\mathrm{CD} 4^{+} \mathrm{T}$ cells and $\mathrm{B}$ cells.

Serum from Aire Ar- $^{-/}$ice with lung disease was shown to contain autoantibodies that bound to the bronchiolar epithelium of frozen lung sections, and the authors purified an $80 \mathrm{kDa}$ protein from lung lysates that is targeted by these autoantibodies in Aire - $^{-/}$but not Aire $^{+/+}$mouse serum. The protein was identified as vomeromodulin, the expression of which is confined almost exclusively to the respiratory epithelium, which confirms that the autoimmune response is lung specific in this model. In keeping with the presence of an autoantibody response to vomeromodulin, there was also a significant increase in the number of interferon- $\gamma$-producing vomeromodulin-specific T cells in the periphery of Aire ${ }^{-/}$compared with $\mathrm{Aire}^{+/+}$mice. The expression of vomeromodulin in purified thymic stroma was shown to be AIRE dependent, which indicates that a defect in central tolerance in Aire ${ }^{-/-}$mice leads to the escape of vomeromodulin-specific $\mathrm{T}$ cells into the periphery, where they can cause damage to the lungs.

The deletion of self-reactive $T$ cells during central tolerance in the thymus is rarely complete and the presence of vomeromodulin-specific $\mathrm{T}$ cells in the periphery of wild-type mice was confirmed by an adjuvant immunization protocol that was sufficient to break peripheral tolerance to vomeromodulin and resulted in a lung-restricted disease. Lung-specific disease could also be induced in mice by the adoptive transfer of vomeromodulin-specific T cells isolated from immunized mice.

Although a direct human homologue of vomeromodulin does not seem to exist, a human patient with APS1 involving lung disease had immunoreactivity to LPLUNC1, which is the adjacent transcript upstream of the human vomeromodulin pseudogene and which has a similar domain structure and expression pattern to vomeromodulin.

This study therefore shows that an autoimmune response to a lung-specific autoantigen is sufficient to trigger ILD both as part of a multiorgan syndrome and as an isolated disease. The development of an inducible autoimmune lung disease model through immunization with vomeromodulin should lead the way to a better understanding of and potential therapy for ILD.

Kirsty Minton

ORIGINAL RESEARCH PAPER Shum, A. K. et al. Identification of an autoantigen demonstrates a link between interstitial lung disease and a defect in central tolerance. Sci. Trans. Med. 1, 9ra20 (2009) 